\title{
Acute Hypoxic Respiratory Failure and Septic Shock Secondary to Mycoplasma Pneumoniae Pneumonia Complicated by Heroin Overdose
}

\author{
Jesse C. Wu ${ }^{1,2}$, Matthew Solomon ${ }^{3}$, Amanda L. Webb McAdams 1, 4 , Latha Ganti 1, 2, 5, 6 \\ 1. Emergency Medicine, University of Central Florida College of Medicine, Orlando, USA 2. Emergency Medicine, \\ Osceola Regional Medical Center, Kissimmee, USA 3. Emergency Medicine, Brown University, Providence, USA 4. \\ Emergency Medicine, Brandon Regional Hospital, Brandon, USA 5. Emergency Medicine, Envision Physician Services, \\ Nashville, USA 6. Emergency Medical Services, Polk County Fire Rescue, Bartow, USA
}

Corresponding author: Latha Ganti, latha.ganti@ucf.edu

\begin{abstract}
We present the case of a woman with a past medical history of intravenous drug use and tobacco abuse who was brought into the emergency department by emergency medical services after a heroin overdose. She was found to be in acute hypoxic respiratory failure and developed septic shock secondary to Mycoplasma pneumonia. In this case report, the presentation and management of fulminant Mycoplasma pneumonia are discussed, along with plain chest radiography findings.
\end{abstract}

Review began 10/13/2020 Review ended 10/15/2020 Published 10/22/2020

\section{๑) Copyright 2020}

Wu et al. This is an open access article distributed under the terms of the Creative Commons Attribution License CC-BY 4.0., which permits unrestricted use, distribution, and reproduction in any medium, provided the original author and source are credited.
Categories: Emergency Medicine, Pulmonology

Keywords: intravenous drug use, tobacco, heroin, hypoxic respiratory failure, septic shock, mycoplasma pneumoniae, pneumonia

\section{Introduction}

Mycoplasma pneumoniae is one of the most common causes of upper respiratory infection (URI), acute bronchitis, and community-acquired pneumonia (CAP) [1]. The infection is transmitted from person-toperson through respiratory droplets and has an average incubation time between two and three weeks. Outbreaks typically occur in people living in close quarters, such as schools, homes, and military barracks [2]. Mycoplasma pneumoniae lacks a cell wall, and therefore it cannot be visualized by gram stain. Additionally, it is not sensitive to antibiotics, such as penicillin, which targets cell wall synthesis. URI and bronchitis are the most common presentation of M. pneumoniae infection. The patient typically presents with cough, rhinorrhea, rhinitis, and pharyngitis [3]. Less commonly, M. pneumoniae can present as CAP with mild fever, headache, malaise, cough, shortness of breath, and pleuritic chest pain. The pneumonia is typically mild and self-limited. Patients usually recover completely even without antibiotics. In rare cases, fulminant pneumonia can develop, resulting in respiratory failure and necessitating pulmonary support, targeted antibiotics therapy, and microbiological diagnosis by polymerase chain reaction, nucleic acid amplification tests, or serology [4].

\section{Case Presentation}

A 34-year-old woman with a past medical history of intravenous (IV) drug use and tobacco abuse was brought to a free-standing emergency department (ED) by the emergency medical services (EMS) for heroin overdose. She was lethargic and was given two doses of naloxone by EMS with some improvement in her level of consciousness. She was given another dose of naloxone in the ED and was put on $2 \mathrm{~L}$ per minute oxygen by nasal cannula due to hypoxia. However, she remained somnolent although arousable. Her oxygen saturation improved to $95 \%$. She was tachycardic with a rate of 114 beats per minute (bpm). Otherwise, she was afebrile, and her blood pressure and respiratory rate were within normal limits. Her white blood cell (WBC) count was normal, and her urine drug screen was positive for opiates, amphetamines, and cocaine. She received $2 \mathrm{~L}$ of normal saline and ceftriaxone prophylaxis against aspiration pneumonia, and was transferred to the main ED. On arrival, she was found to be febrile $\left(102^{\circ} \mathrm{F}\right)$ and tachycardic. She received another bolus of normal saline and one more dose of naloxone with mild improvement in her mental status. The following morning she desaturated and her heart rate increased to $140 \mathrm{bpm}$. She was placed on a nonrebreather mask with improvement in her oxygen saturation. Chest radiograph performed at the time showed bilateral airspace opacities, suggestive of pneumonia (Figure 1). 


\section{Cureus}

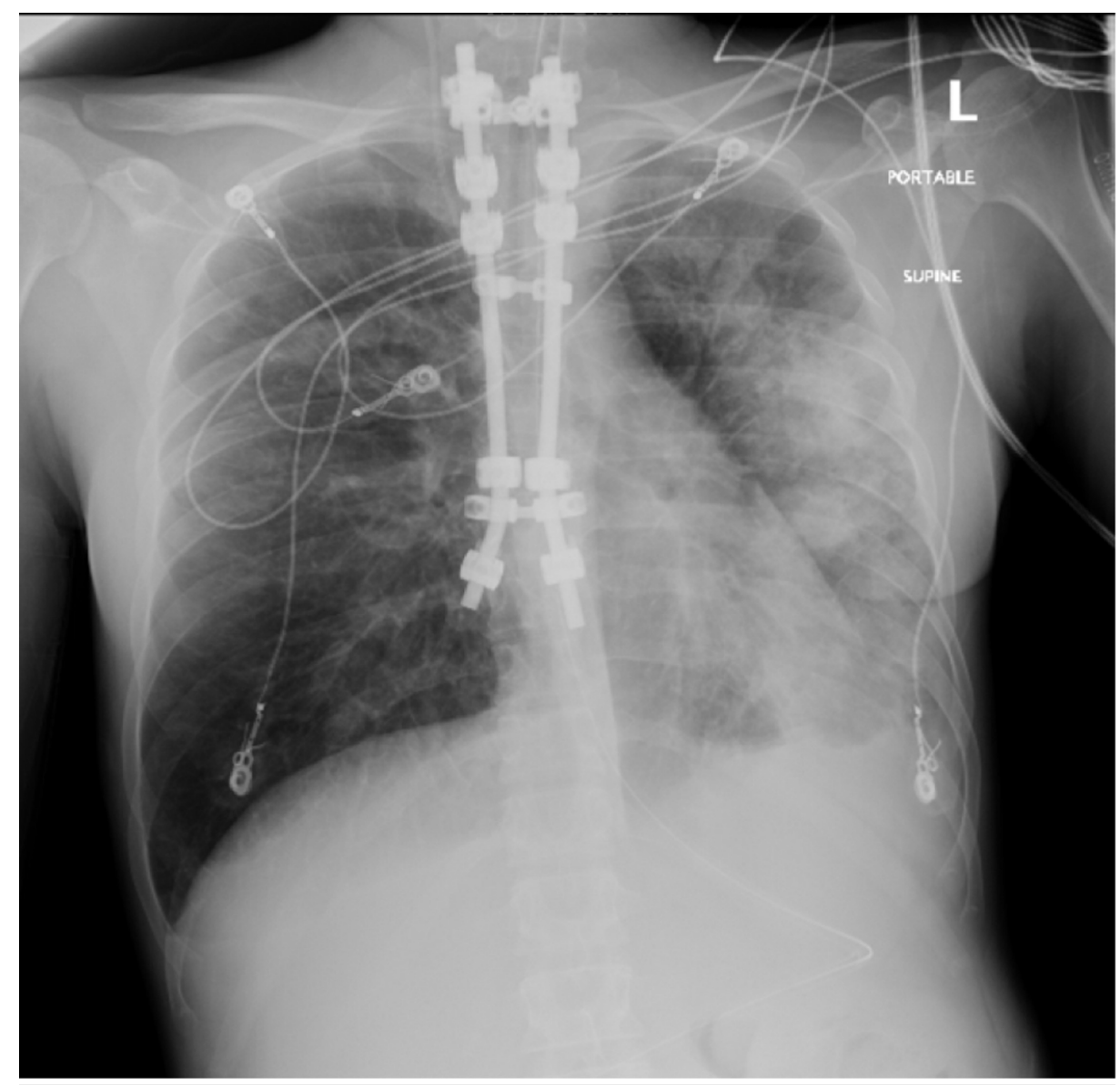

FIGURE 1: Hospital day 1: multiple patchy opacities in the left lung, representing pneumonia. A small left pleural effusion is also present.

However, she desaturated again to $80 \%$ and was found to be hypotensive to $88 / 44 \mathrm{mmHg}$. She was responsive to pain only. Rapid response was called, and the patient was intubated for airway protection and acute hypoxic respiratory failure. She was then transferred to the intensive care unit (ICU) for further care and management. The patient was mechanically ventilated and was started on vancomycin and piperacillintazobactam, IV fluid support, and a norepinephrine infusion. Her lactic acid was $1.8 \mathrm{mmol} / \mathrm{L}$, procalcitonin was $44.04 \mathrm{ng} / \mathrm{mL}$, and WBC count was $13.97 \times 10^{3}$. She remained stable in the ICU and was gradually weaned off the ventilator and pressor. She was extubated the next morning. On hospital day 2 , she still required IV fluid support to maintain mean arterial pressure (MAP) above $65 \mathrm{mmHg}$, and she was still tachycardic at 112 bpm. Otherwise, her fever resolved and she was saturating normally on 5 LPM oxygen by nasal cannula. She had bilateral rales and rhonchi on examination. Her leukocytosis resolved, but her procalcitonin increased to $120.60 \mathrm{ng} / \mathrm{mL}$. Her repeat chest radiograph showed worsening consolidation bilaterally, and her $M$. pneumoniae IgM was positive (Figure 2). 


\section{Cureus}

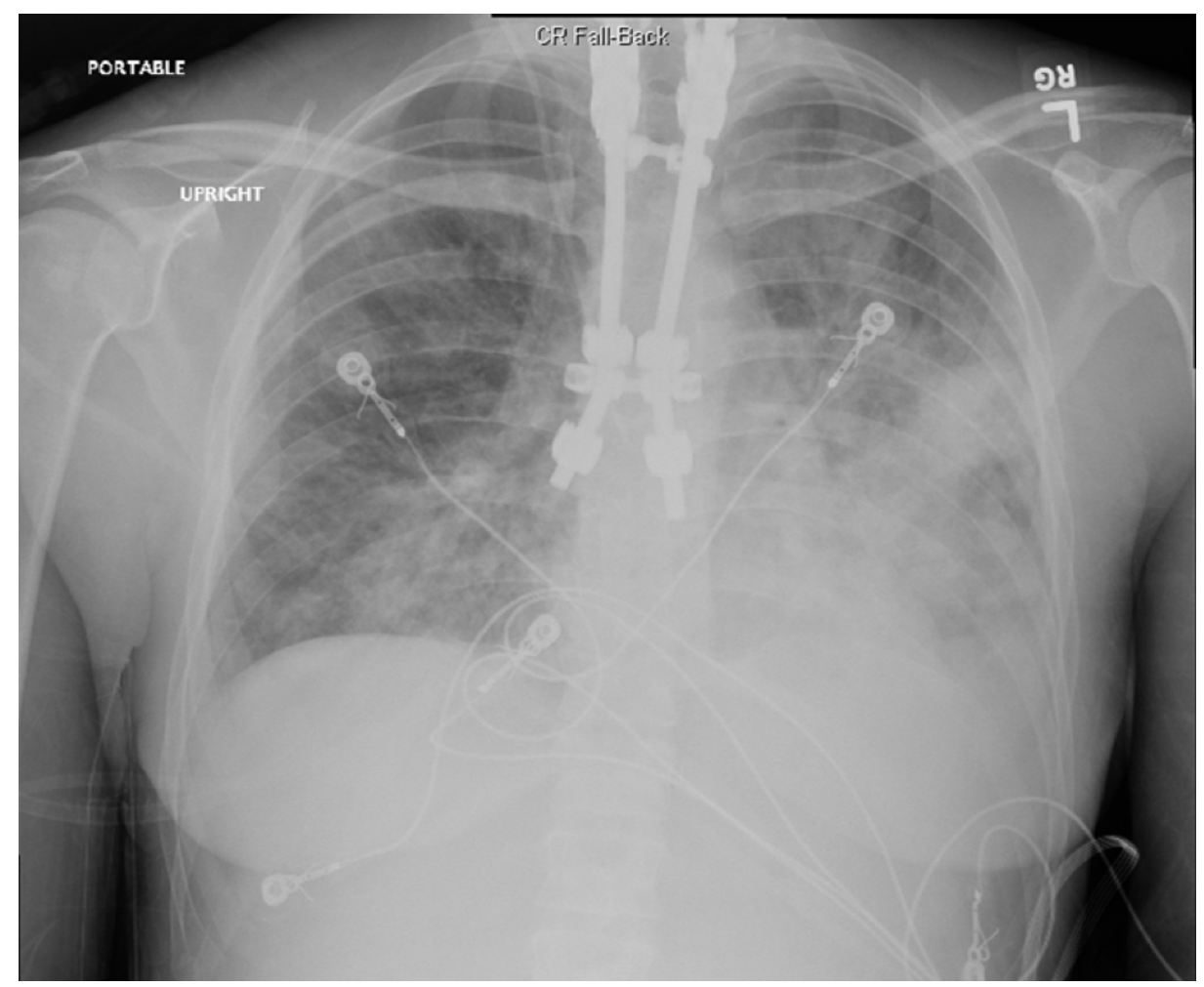

FIGURE 2: Hospital day 2: worsening of the left lung field airspace opacities and right lower lobe airspace opacity, representing interval worsening of bilateral pneumonia.

She was started on azithromycin for presumptive bilateral M. pneumoniae pneumonia (MPP). Further workup revealed that she was human immunodeficiency virus (HIV) negative. Urine antigens were negative for Streptococcus or Legionella. Sputum cultures and urinalysis were additionally negative for any bacteria. However, the patient was found to have chronic hepatitis B (hepatitis B surface antigen and core IgG positive, surface antibody and core IgM negative). Transthoracic echocardiogram revealed no vegetations, and the ejection fraction was 65-75\%. On hospital day 3, her blood pressure improved, with an MAP of 80$90 \mathrm{mmHg}$. She was gradually weaned off nasal cannula, maintained a normal oxygen saturation, and her heart rate normalized throughout the day. Her repeat procalcitonin was $66.32 \mathrm{ng} / \mathrm{mL}$. On hospital day 4 , vancomycin and piperacillin-tazobactam were discontinued due to negative workup for other sources of infection. The patient continued to improve on hospital day 5 with interval decreased in bilateral pneumonia on chest X-ray (Figure 3). 


\section{Cureus}

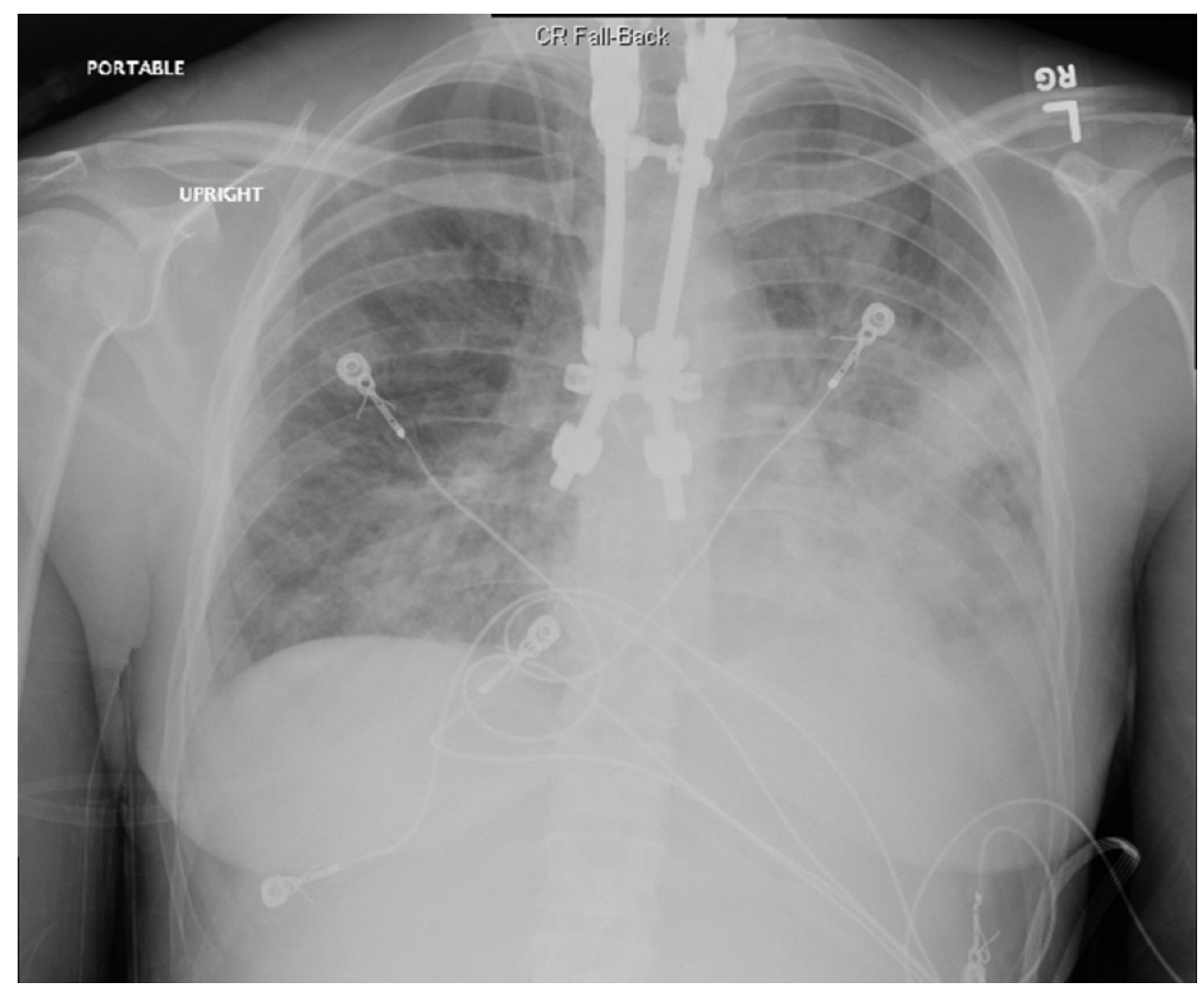

FIGURE 3: Chest radiograph on hospital day 5 showing interval decrease in bilateral airspace opacities, representing an improvement in bilateral pneumonia.

She was eventually discharged in stable condition on hospital day 7, after a five-day course of azithromycin.

\section{Discussion}

MPP infection is typically mild; however, in rare cases, fulminant infection with respiratory failure can result, especially in young adults. Fulminant cases are estimated to be $0.5-2 \%$ of all MPP cases, with mortality reported to be 3-5\% in the 1980s [4]. Clinical features include fever, cough, dyspnea, and bilateral infiltrates and pleural effusion on chest X-ray. Laboratory findings include elevated WBC count, C-reactive protein, lactate dehydrogenase, aspartate transaminase, and alanine transaminase. First-line treatment for MPP includes macrolides (e.g. azithromycin) with tetracyclines (e.g. doxycycline). Second-line agents include respiratory fluoroquinolones (e.g. levofloxacin or moxifloxacin), which are reserved for macrolide-resistant cases [5].

To date, there are several reported cases of fulminant MPP resulting in respiratory failure [6-7]. However, there are no reports of MPP with respiratory failure complicated by opiate overdose. In our case presentation, the patient presented with somnolence, dyspnea, tachycardia, and fever in the setting of heroin overdose. The differential diagnosis can be broad, including alcohol withdrawal, thyroid storm, anticholinergic or serotonergic toxicity, infection, and multiple drug overdose. It was important to explore the numerous causes of her symptoms and not just anchor on the drug overdose as the only cause of her symptoms. Workup should include blood cultures to look for bloodstream infection, urinalysis to look for urinary tract infection, chest X-ray to look for pneumonia, laboratory evaluation of alcohol level, coingestions, thyroid hormone, and respiratory pathogens, skin examination to check for cellulitis or abscesses, HIV testing, and echocardiogram to evaluate for vegetations from infective endocarditis given her IV drug use history.

The current case describes a rare presentation of MPP complicated by opiate overdose, requiring intubation, mechanical ventilation, targeted antibiotics, IV fluid and pressor support. This case highlights the importance of thorough physical examination and workup of undifferentiated complaints with a broad differential diagnosis in mind.

\section{Conclusions}

Patients with MPP and respiratory failure should be monitored closely, with priority given to airway, breathing, and circulation. Targeted antibiotics therapy is the first-line treatment, with consideration of 


\section{Additional Information}

\section{Disclosures}

Human subjects: Consent was obtained by all participants in this study. HCA Centralized Algorithms for Research Rules on IRB Exemptions (CARRIE)/IRB manager issued approval 2020-460. HCA Centralized Algorithms for Research Rules on IRB Exemptions (CARRIE)/IRB manager issued approval [2020-460]. Based on the information provided and attested as true, the research plan described does not require IRB oversight. This is because you are either a) not engaging in research with human subjects as defined by federal regulations; b) engaging in research with human subjects deemed excluded from IRB oversight per 45CFR46.102(l) OR c) engaging in research with sufficient human subject protections in the design to meet one or more IRB exemption criteria set forth in 45CFR46.104. Conflicts of interest: In compliance with the ICMJE uniform disclosure form, all authors declare the following: Payment/services info: All authors have declared that no financial support was received from any organization for the submitted work. Financial relationships: All authors have declared that they have no financial relationships at present or within the previous three years with any organizations that might have an interest in the submitted work. Other relationships: All authors have declared that there are no other relationships or activities that could appear to have influenced the submitted work.

\section{Acknowledgements}

This research was supported (in whole or in part) by HCA Healthcare and/or an HCA Healthcare affiliated entity. The views expressed in this publication represent those of the author(s) and do not necessarily represent the official views of HCA Healthcare or any of its affiliated entities.

\section{References}

1. Foy HM, Kenny GE, Cooney MK, Allan ID: Long-term epidemiology of infections with Mycoplasma pneumoniae. J Infect Dis. 1979, 139:681-687. 10.1093/infdis/139.6.681

2. Clyde WA Jr: Clinical overview of typical Mycoplasma pneumoniae infections. Clin Infect Dis. 1993, 17:3236.

3. Mansel JK, Rosenow EC 3rd, Smith TF, Maryin JW: Mycoplasma pneumoniae pneumonia. Chest. 1989, 95:639-646. 10.1378/chest.95.3.639

4. Chan ED, Welsh CH: Fulminant Mycoplasma pneumoniae pneumonia. West J Med. 1995, 162:133-142.

5. Izumikawa K: Clinical features of severe or fatal Mycoplasma pneumoniae pneumonia . Front Microbiol. 2016, 7:800. 10.3389/fmicb.2016.00800

6. Takiguchi Y, Shikama N, Aotsuka N, Koseki H, Terano T, Hirai A: Fulminant Mycoplasma pneumoniae pneumonia. Intern Med. 2001 , 40:345-348. 10.2169/internalmedicine.40.345

7. Chaudhry R, Tabassum I, Kapoor L, Chhabra A, Sharma N, Broor S: A fulminant case of acute respiratory distress syndrome associated with Mycoplasma pneumoniae infection. Indian J Pathol Microbiol. 2010, 53:555-557. 10.4103/0377-4929.68283 